\title{
Iterative Detection and Decoding Using Approximate Bayesian Theorem Based PDA Method Over MIMO Nakagami- $m$ Fading Channels
}

\author{
Shaoshi Yang ${ }^{\dagger *}$ and Lajos Hanzo ${ }^{\dagger}$ \\ ${ }^{\dagger}$ School of Electronics and Computer Science, University of Southampton, Southampton, SO17 1BJ, UK \\ *School of Information and Communication Engineering, Beijing University of Posts and Telecommunications, 100876, Beijing, China \\ Email: $\{$ sy7g09, lh\}@ecs.soton.ac.uk, http://www-mobile.ecs.soton.ac.uk
}

\begin{abstract}
In this paper, the design of iterative detection and decoding (IDD) schemes relying on a low-complexity probabilistic data association (PDA) aided method is conceived for turbo-coded multiple-input multiple-output (MIMO) systems communicating over Nakagami- $m$ fading channels. The known PDA based MIMO detectors typically operate purely in the probabilitydomain. We show that the classic relationship where the extrinsic LLRs are given by subtracting the $a$ priori LLRs from the $a$ posteriori LLRs does not hold for the existing PDA based MIMO detectors. Therefore, the PDA method is not readily applicable to the IDD receiver. To overcome this predicament, we propose an approximate Bayesian theorem based log-domain PDA (AB-LogPDA) detector, as well as a novel simple approach of calculating the bit-wise extrinsic LLRs for the AB-Log-PDA, which makes the AB-Log-PDA well-suited for employment in IDD receivers. It is shown that the proposed AB-Log-PDA based IDD scheme is capable of achieving a comparable performance to that of the optimal maximum a posteriori (MAP) detector based IDD receiver, while imposing a much lower computational complexity in the scenarios considered.
\end{abstract}

Index Terms-Extrinsic information transfer (EXIT) chart, iterative detection and decoding, multiple-input multiple-output (MIMO), probabilistic data association (PDA), Nakagami- $m$ fading channel

\section{INTRODUCTION}

Iterative detection and decoding (IDD) is capable of achieving a near-optimum performance at a significantly lower complexity than the optimal joint detector/decoder [1]. Even so, the computational complexity imposed by the IDD might remain the limiting factor in practical applications.

The Probabilistic Data Association (PDA) method is a reduced-complexity design alternative of maximum a posteriori (MAP) decoders/detectors/equalizers [2]-[7]. As an efficient interference-modelling process, the key feature of PDA is the repeated conversion of a multimodal Gaussian mixture probability to a single multivariate Gaussian distribution. Therefore, the accuracy of the Gaussian approximation dominates the attainable performance. In uncoded MIMO systems using quadrature amplitude modulation (QAM), the quality of the Gaussian approximation in PDA may be improved by transforming the symbol-based model into a bit-based model,

The financial support of the China Scholarship Council (CSC), of the Reserach Councils UK (RCUK) under the India-UK Advanced Technology Centre (IU-ATC), and of the EU under the auspices of the Concerto project is gratefully acknowledged. which in effect increases the length of the effective transmitted signal vector by the number of bits per symbol, and reduces the effective constellation to a binary constellation [6]. Regarding improving the quality of the Gaussian approximation in FEC coded MIMO systems, we benefit from having an increased degree of freedom to exploit. For example, the soft information gleaned from the output of the FEC decoder via feedback is deemed to be a more reliable information source than the raw received signal at the fading channel's output, and therefore it may reduce the bias in modelling the inter-antenna interference (IAI) components.

Against this background, in this paper we aim for designing a low-complexity IDD scheme relying on the PDA method for FEC coded MIMO systems using $M$-QAM for transmission over Nakagami- $m$ fading channels. However, there are further particular challenges that render the IDD design using PDA less straightforward than it seems to be. Firstly, to the best of our knowledge, all the existing PDA detectors conceived for uncoded systems [2]-[7] operate purely in the probabilitydomain, which results in a poor numerical stability and low accuracy in IDD scenarios and hence leads to a degraded performance. By contrast, in this paper we propose a new approximate Bayesian theorem based log-domain PDA (ABLog-PDA) MIMO detector, which is better suited for the IDD scheme. Furthermore, for the existing family of PDA methods [2]-[7] as well as the proposed AB-Log-PDA, it is unclear how to produce the "correct" extrinsic log-likelihood ratios (LLRs). required by the concatenated outer FEC decoder. A natural way of generating the bit-wise extrinsic LLRs is to subtract the bit-wise a priori LLRs from the bit-wise $a$ posteriori LLRs generated from the estimated symbol-wise APPs of the PDA. However, our study demonstrate that the symbol-wise APPs produced by the approximate Bayesian theorem based PDA detector are not the "true" APPs, but rather some sort of "nominal" APPs, for which the classic relationship where the a posteriori LLRs are given by the sum of the a priori LLRs and the extrinsic LLRs does not hold. Nonetheless, the symbol-wise probabilities output by the PDA based methods have been referred to as APPs [2]-[7], and the distinctions between these "nominal" APPs and the true APPs have never been reported before. Therefore, a novel approach of producing the bit-wise extrinsic LLRs for the proposed ABLog-PDA is presented, which makes it possible to design a 


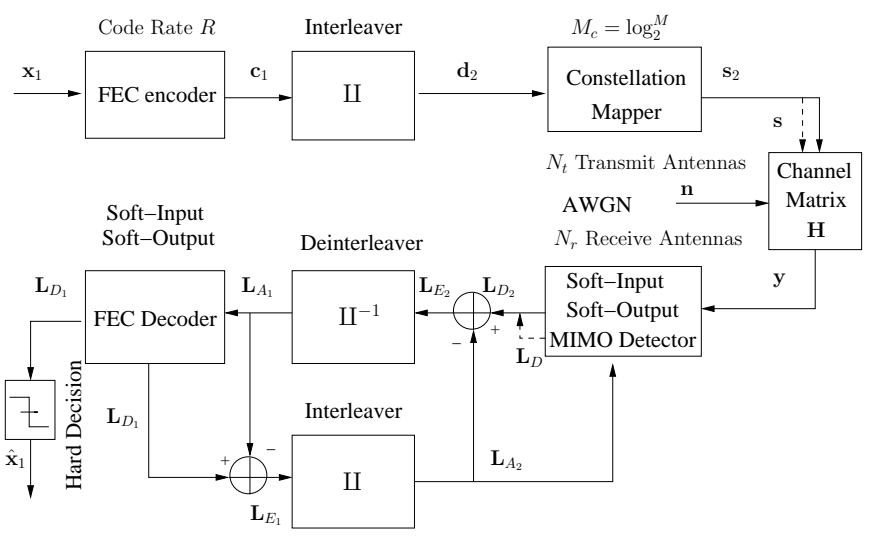

Fig. 1. FEC coded MIMO system with IDD receiver. The subscript "1" denotes the modules associated with the "outer" FEC encoder/decoder, and the subscript " 2 " denotes the modules that are connected with the "inner" space-time mapper/detector. The arrow with dashed line indicates that $\mathbf{s}$ and $\mathbf{L}_{D}$ are the subvectors of $\mathbf{s}_{2}$ and $\mathbf{L}_{D_{2}}$, respectively.

low-complexity IDD scheme based on the proposed AB-LogPDA. Notably, the proposed IDD scheme is shown to strike an attractive performance versus complexity tradeoff compared to the optimum MAP-based IDD scheme. For example, in some scenarios the performance of the proposed AB-Log-PDA based IDD scheme is within $0.5 \mathrm{~dB}$ or even less of that of the MAP-based IDD scheme, while imposing a significantly lower complexity.

\section{SYSTEM MODEL}

We consider the FEC coded MIMO system of Fig. 1. At the transmitter, the $\left(L_{f} \times 1\right)$ source-bit frame $\mathbf{x}_{1}$ is firstly encoded by a rate $R<1$ FEC encoder (typically a convolutional code, a turbo code or an LDPC code) into the $\left(\frac{L_{f}}{R} \times 1\right)$ coded-bit frame $\mathbf{c}_{1}$. In order to guard against bursty fading, $\mathbf{c}_{1}$ is then passed through a bit-interleaver. Then the $\left(\frac{L_{f}}{R} \times 1\right)$ interleaver output bit-frame $\mathbf{d}_{2}$ is mapped to the $\left(\frac{L_{f}}{R M_{b}} \times 1\right)$ symbol-frame $\mathbf{s}_{2}$, with each symbol taken from the modulation constellation $\mathcal{A}=\left\{a_{1}, a_{2}, \cdots, a_{M}\right\}$, where $M_{b}=\log _{2}^{M}$ is the number of bits per constellation symbol. Finally, $\mathbf{s}_{2}$ is transmitted in form of the $\left(N_{t} \times 1\right)$ "vector of symbols" $\mathrm{s}$ by $N_{t} \geq 1$ transmit antennas per channel use. At the output of the fading channel $\mathbf{H}$, the received $\left(N_{r} \times 1\right)$-element complex-valued baseband signal vector per channel use is represented by

$$
\mathbf{y}=\mathbf{H s}+\mathbf{n},
$$

where $\mathbf{s}=\left[s_{1}, s_{2}, \cdots, s_{N_{t}}\right]^{T}$ is normalized by the componentwise energy constraint $\mathbb{E}\left(\left\|s_{i}\right\|^{2}\right)=E_{\mathbf{s}} / N_{t}$ in order to maintain a total transmit power $E_{\mathbf{s}}$ per channel use; and $\mathbf{n}$ is the $\left(N_{r} \times 1\right)$-element zero-mean complex-valued Gaussian noise vector with a covariance matrix of $2 \sigma^{2} \mathbf{I}_{N_{r}}$ where $\mathbf{I}_{N_{r}}$ represents an $\left(N_{r} \times N_{r}\right)$-element identity matrix; and $\mathbf{H}$ is an $\left(N_{r} \times N_{t}\right)$-element complex-valued matrix with entries $h_{j i}$ perfectly known to the receiver, $j=1, \cdots, N_{r}, i=1, \cdots, N_{t}$. In this paper, we assume that $h_{j i}=r \exp (j \theta)$ is independent and identically distributed (i.i.d), the phase $\theta$ is uniformly distributed and independent of the envelope $r$, while $r$ obeys Nakagami- $m$ distribution having the probability density func- tion (PDF) of [8]

$$
p(r)=\frac{2}{\Gamma(m)}\left(\frac{m}{\Omega}\right)^{m} r^{2 m-1} \exp \left(-m r^{2} / \Omega\right), r \geq 0,
$$

where $\Gamma(\cdot)$ represents the Gamma function, $\Omega \triangleq \mathbb{E}\left(r^{2}\right)$, and the Nakagami fading parameter is $m \triangleq \Omega^{2} / \mathbb{E}\left[\left(r^{2}-\Omega\right)^{2}\right]$, $m \geq 0.5$. Note that the Nakagami- $m$ fading model captures a wide range of realistic fading environments, encompassing the most frequently used Rayleigh and Ricean fading models as special cases. More specifically, the parameter $m$ indicates the severity of the fading. As $m$ becomes smaller, the fading effects become more severe. For example, when $m$ decreases to 0.5, Eq. (2) approaches the one-sided Gaussian distribution; when $m=1$, Eq. (2) reduces to a Rayleigh PDF, and as $m \rightarrow \infty$, Eq. (2) reduces to a $\delta$-distribution located at $r=1$, which corresponds to imposing no fading on the amplitude of the transmitted signal, but only a "pure random phase" obeying a uniform distribution on the circle of radius $\sqrt{\Omega}$.

\section{The Proposed AB-Log-PDA Relying ON A Priori SOFT FEEDBACK FROM THE FEC DECODER}

The original received signal model of (1) may be rewritten as

$$
\mathbf{y}=s_{i} \mathbf{h}_{i}+\underbrace{\sum_{k \neq i} s_{k} \mathbf{h}_{k}}_{\mathbf{u}_{i}}+\mathbf{n} \triangleq s_{i} \mathbf{h}_{i}+\underbrace{\mathbf{u}_{i}+\mathbf{n}}_{\mathbf{v}_{i}},
$$

where $\mathbf{h}_{i}$ denotes the $i$-th column of $\mathbf{H}$, and $s_{i}$ is the $i$-th symbol of $\mathbf{s}$, while $\mathbf{u}_{i}$ is the sum of $\left(N_{t}-1\right)$ IAI components contaminating the symbols $s_{i}, i, k=1,2, \cdots, N_{t}$, and $\mathbf{v}_{i}$ is the interference-plus-noise term for $s_{i}$. The AB-Log-PDA method uses the received signal $\mathbf{y}$, the channel matrix $\mathbf{H}$, as well as the a priori soft information as its input parameters, and outputs the estimated a posteriori probabilities (APPs) of the transmitted symbols $\left\{s_{i}\right\}_{i=1, \cdots, N_{t}}$. For the sake of improving the numerical stability and accuracy, some of the key procedures are implemented in the log-domain.

As mentioned before, each symbol $s_{i}$ to be detected in Eq. (3) is contaminated by both the noise $\mathbf{n}$ and the interfering signal $\mathbf{u}_{i}$. The noise is generally undesirable, but the interference might potentially become a useful signal, which is only not desired at some specific instant. Hence, if we can predict or estimate the interfering symbols $\left\{s_{k}\right\}_{k \neq i}$, the performance degradation caused by $\mathbf{u}_{i}$ may be mitigated, or even completely eliminated under idealized conditions, such that a receive diversity order of $N_{r}$ is approached. Although initially we do not have any a priori knowledge about the distribution of $\left\{s_{k}\right\}_{k \neq i}$, we know exactly the distribution of the noise $\mathbf{n}$ and are also aware of the legitimate values of $\left\{s_{k}\right\}_{k \neq i}$. Hence it is realistic to generate a coarse estimate of $s_{i}$ relying solely on our knowledge of the noise distribution and the original modulation constellation $\mathcal{A}$ employed at the beginning. Therefore, we may assume that the interferenceplus-noise term $\mathbf{v}_{\mathbf{i}}$ obeys a single $N_{r}$-variate Gaussian distribution. In order to fully characterize the complex random vector $\mathbf{v}_{\mathbf{i}}$, which is not necessarily proper [5], [9], [10], we 
TABLE I

Probabilities Computed in ONE ITERATION BETWEen THE AB-LOG-PDA DETECTOR AND THE FEC DECODER

\begin{tabular}{c|cccccc}
\hline & 1 & 2 & $\cdots$ & $m$ & $\cdots$ & $M$ \\
\hline $\mathbf{P}^{(z)}(1)$ & $P_{1}^{(z)}\left(s_{1} \mid \mathbf{y}\right)$ & $P_{2}^{(z)}\left(s_{1} \mid \mathbf{y}\right)$ & $\cdots$ & $P_{m}^{(z)}\left(s_{1} \mid \mathbf{y}\right)$ & $\cdots$ & $P_{M}^{(z)}\left(s_{1} \mid \mathbf{y}\right)$ \\
$\mathbf{P}^{(z)}(2)$ & $P_{1}^{(z)}\left(s_{2} \mid \mathbf{y}\right)$ & $P_{2}^{(z)}\left(s_{2} \mid \mathbf{y}\right)$ & $\cdots$ & $P_{m}^{(z)}\left(s_{2} \mid \mathbf{y}\right)$ & $\cdots$ & $P_{M}^{(z)}\left(s_{2} \mid \mathbf{y}\right)$ \\
$\vdots$ & $\vdots$ & $\vdots$ & $\cdots$ & $\vdots$ & $\cdots$ & $\vdots$ \\
$\mathbf{P}^{(z)}(i)$ & $P_{1}^{(z)}\left(s_{i} \mid \mathbf{y}\right)$ & $P_{2}^{(z)}\left(s_{i} \mid \mathbf{y}\right)$ & $\cdots$ & $P_{m}^{(z)}\left(s_{i} \mid \mathbf{y}\right)$ & $\cdots$ & $P_{M}^{(z)}\left(s_{i} \mid \mathbf{y}\right)$ \\
$\vdots$ & $\vdots$ & $\vdots$ & $\cdots$ & $\vdots$ & $\cdots$ & $\vdots$ \\
$\mathbf{P}^{(z)}\left(N_{t}\right)$ & $P_{1}^{(z)}\left(s_{N_{t}} \mid \mathbf{y}\right)$ & $P_{2}^{(z)}\left(s_{N_{t}} \mid \mathbf{y}\right)$ & $\cdots$ & $P_{m}^{(z)}\left(s_{N_{t}} \mid \mathbf{y}\right)$ & $\cdots$ & $P_{M}^{(z)}\left(s_{N_{t}} \mid \mathbf{y}\right)$ \\
\hline
\end{tabular}

specify the mean as

$$
\boldsymbol{\mu}_{i} \triangleq \mathbb{E}\left(\mathbf{v}_{\mathbf{i}}\right)=\sum_{k \neq i} \mathbb{E}\left(s_{k}\right) \mathbf{h}_{k}
$$

the covariance as

$$
\boldsymbol{\Upsilon}_{i} \triangleq \mathbb{C}\left(\mathbf{v}_{i}\right)=\sum_{k \neq i} \mathbb{C}\left(s_{k}\right) \mathbf{h}_{k} \mathbf{h}_{k}^{H}+2 \sigma^{2} \mathbf{I}_{N_{r}},
$$

and the pseudo-covariance as

$$
\underline{\Upsilon}_{i} \triangleq \mathbb{C}_{p}\left(\mathbf{v}_{i}\right)=\sum_{k \neq i} \mathbb{C}_{p}\left(s_{k}\right) \mathbf{h}_{k} \mathbf{h}_{k}^{T}
$$

For each symbol $s_{i}, i=1, \cdots, N_{t}$, we define an $M$ element symbol probability vector $\mathbf{P}^{(z)}(i)$, whose $m$-th element $P_{m}^{(z)}\left(s_{i} \mid \mathbf{y}\right) \triangleq P^{(z)}\left(s_{i}=a_{m} \mid \mathbf{y}\right)$ is the estimate of the APP that we have $s_{i}=a_{m}$ at the $z$-th iteration between the "inner" AB-Log-PDA and the "outer" soft FEC decoder, where $z$ is a nonnegative integer, and $m=1, \cdots, M$. For clarity, these probabilities are explicitly presented in form of the probability-matrix of Table I. Then we have

$$
\begin{gathered}
\mathbb{E}\left(s_{k}\right)=\sum_{m=1}^{M} a_{m} P^{(z)}\left(s_{k}=a_{m} \mid \mathbf{y}\right), \\
\mathbb{C}\left(s_{k}\right)=\sum_{m=1}^{M}\left(a_{m}-\mathbb{E}\left(s_{k}\right)\right)\left(a_{m}-\mathbb{E}\left(s_{k}\right)\right)^{*} P^{(z)}\left(s_{k}=a_{m} \mid \mathbf{y}\right),
\end{gathered}
$$

and

$$
\mathbb{C}_{p}\left(s_{k}\right)=\sum_{m=1}^{M}\left(a_{m}-\mathbb{E}\left(s_{k}\right)\right)^{2} P^{(z)}\left(s_{k}=a_{m} \mid \mathbf{y}\right),
$$

where the pseudo-covariance of a complex random vector $\mathrm{x}$ is defined as [9]

$$
\mathbb{C}_{p}(\mathbf{x}) \triangleq \mathbb{E}\left[(\mathbf{x}-\mathbb{E}(\mathbf{x}))(\mathbf{x}-\mathbb{E}(\mathbf{x}))^{T}\right] .
$$

Note that Eq. (4) - Eq. (9) effectively use all $\left\{\mathbf{P}^{(z)}(k)\right\}_{k \neq i}$ associated with the interfering signal $\left\{s_{k}\right\}_{k \neq i}$ to model $\mathbf{v}_{i}$. Since we do not have any outer a priori knowledge about the distribution of $s_{i} \mid \mathbf{y}$ at the beginning, an all-zero LLR vector will be provided as the input to the AB-Log-PDA. This allzero LLR vector is equivalent to initializing $P^{(z)}\left(s_{i}=a_{m} \mid \mathbf{y}\right)$ with a uniform distribution, i.e.

$$
P^{(z)}\left(s_{i}=a_{m} \mid \mathbf{y}\right)=P^{(z)}\left(s_{i}=a_{m}\right)=\frac{1}{M},
$$

where $z=0, \forall i=1, \cdots, N_{t}$ and $\forall m=1, \cdots, M$.

Based on the assumption that $\mathbf{v}_{i}$ obeys the Gaussian distribution, $\mathbf{y} \mid s_{i}$ is also Gaussian distributed. Let us now define

$$
\mathbf{w} \triangleq \mathbf{y}-s_{i} \mathbf{h}_{i}-\sum_{k \neq i} \mathbb{E}\left(s_{k}\right) \mathbf{h}_{k}
$$

and

$$
\beta_{m, i}^{(z+1)} \triangleq-\left[\begin{array}{c}
\Re(\mathbf{w}) \\
\Im(\mathbf{w})
\end{array}\right]^{T} \boldsymbol{\Lambda}_{i}^{-1}\left[\begin{array}{l}
\Re(\mathbf{w}) \\
\Im(\mathbf{w})
\end{array}\right],
$$

in which the composite covariance matrix $\boldsymbol{\Lambda}_{i}$ is defined as [5]

$$
\boldsymbol{\Lambda}_{i} \triangleq\left[\begin{array}{cc}
\Re\left(\mathbf{\Upsilon}_{i}+\underline{\mathbf{\Upsilon}}_{i}\right) & -\Im\left(\mathbf{\Upsilon}_{i}-\underline{\mathbf{\Upsilon}}_{i}\right) \\
\Im\left(\mathbf{\Upsilon}_{i}+\underline{\mathbf{\Upsilon}}_{i}\right) & \Re\left(\mathbf{\Upsilon}_{i}-\underline{\mathbf{\Upsilon}}_{i}\right)
\end{array}\right],
$$

where $\Re(\cdot)$ and $\Im(\cdot)$ represent the real and imaginary part of a complex variable, respectively. Then the likelihood function of $\mathbf{y} \mid s_{i}=a_{m}$ at the $(z+1)$-st iteration satisfies

$$
p^{(z+1)}\left(\mathbf{y} \mid s_{i}=a_{m}\right) \propto \exp \left(\beta_{m, i}^{(z+1)}\right) .
$$

Upon invoking an approximate form of the Bayesian theorem, the estimated APP of symbol $s_{i}$ at the $(z+1)$-st iteration may be calculated in the probability-domain as

$$
\begin{aligned}
P^{(z+1)}\left(s_{i}=a_{m} \mid \mathbf{y}\right) & \approx \frac{p^{(z+1)}\left(\mathbf{y} \mid s_{i}=a_{m}\right)}{\sum_{m=1}^{M} p^{(z+1)}\left(\mathbf{y} \mid s_{i}=a_{m}\right)} \\
& =\frac{\exp \left(\beta_{m, i}^{(z+1)}-\gamma\right)}{\sum_{m=1}^{M} \exp \left(\beta_{m, i}^{(z+1)}-\gamma\right)}
\end{aligned}
$$

where $\gamma \triangleq \max _{m=1, \cdots, M} \beta_{m, i}^{(z+1)}$. In order to further improve the achievable numerical stability and accuracy, the log-domain form of (16) is formulated as

$$
\begin{aligned}
\psi_{m, i}^{(z+1)} & \triangleq \ln \left(P^{(z+1)}\left(s_{i}=a_{m} \mid \mathbf{y}\right)\right) \\
& =\left(\tilde{\beta}_{m, i}^{(z+1)}\right)-\ln \left(\sum_{m=1}^{M} \exp \left(\tilde{\beta}_{m, i}^{(z+1)}\right)\right),
\end{aligned}
$$

in which we have $\tilde{\beta}_{m, i}^{(z+1)} \triangleq \beta_{m, i}^{(z+1)}-\gamma$, and the second term of the right-hand-side expression may be computed by invoking the "Jacobian logrithm" of [11]. Upon invoking the Max-log approximation, (17) may be further simplified as

$$
\psi_{m, i}^{(z+1)}=\tilde{\beta}_{m, i}^{(z+1)}-\max _{m=1, \cdots, M} \tilde{\beta}_{m, i}^{(z+1)} .
$$

As a result, the estimated symbol-wise APP of $s_{i}$ is given by

$$
P^{(z+1)}\left(s_{i}=a_{m} \mid \mathbf{y}\right) \approx e^{\psi_{m, i}^{(z+1)}},
$$

which will replace the value of $P^{(z)}\left(s_{i}=a_{m} \mid \mathbf{y}\right)$ in Table I. These updated symbol-wise APPs have to be converted to equivalent bit-wise LLRs, of which the extrinsic parts will be delivered to the outer FEC decoder of Fig. 1. Then, the extrinsic LLRs output by the FEC decoder will be converted to symbol-wise probabilities in the next iteration for the sake of generating new estimates of the symbol-wise APPs using the AB-Log-PDA. For reasons of explicit clarity, the proposed 
TABLE II

SUMmary OF THE AB-LOG-PDA Algorithm

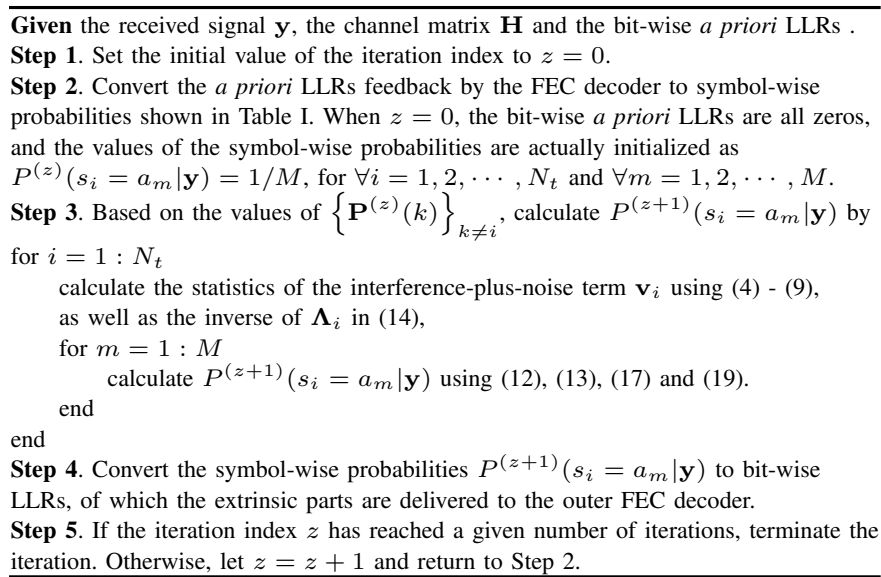

AB-Log-PDA algorithm is summarized in Table II.

\section{EXTRINSIC LLR CALCULATION FOR AB-LOG-PDA IN FEC-CODED MIMO SYSTEMS}

In a FEC-coded MIMO system employing the IDD scheme of Fig. 1, typically the extrinsic LLRs of the coded bits are exchanged between the soft-input soft-output (SISO) MIMO detector and the SISO FEC decoder. Therefore, in order to employ the AB-Log-PDA in the IDD scheme, the AB-LogPDA has to output the correct extrinsic LLRs of the FEC coded bits, which is however, not quite as straightforward as it seems at first sight.

Denote the $l$-th bit of the $i$-th symbol $s_{i}$ as $b_{i l}$, then the $a$ posteriori LLR of $b_{i l}$ based on the true symbol-wise APPs of $P\left(s_{i}=a_{m} \mid \mathbf{y}\right)$ may be written as

$$
\begin{aligned}
L_{D}\left(b_{i l} \mid \mathbf{y}\right) & =\ln \frac{P\left(b_{i l}=+1 \mid \mathbf{y}\right)}{P\left(b_{i l}=-1 \mid \mathbf{y}\right)} \\
& =\ln \frac{\sum_{\forall a_{m} \in \mathcal{A}_{l}^{+}} P\left(s_{i}=a_{m} \mid \mathbf{y}\right)}{\sum_{\forall a_{m} \in \mathcal{A}_{l}^{-}} P\left(s_{i}=a_{m} \mid \mathbf{y}\right)} \\
& =L_{E}\left(b_{i l} \mid \mathbf{y}\right)+\underbrace{\ln \frac{P\left(b_{i l}=+1\right)}{P\left(b_{i l}=-1\right)}}_{L_{A}\left(b_{i l}\right)} .
\end{aligned}
$$

In other words, we have

$$
\begin{aligned}
L_{E}\left(b_{i l} \mid \mathbf{y}\right)=\ln \frac{\sum_{\forall a_{m} \in \mathcal{A}_{l}^{+}} P\left(s_{i}=a_{m} \mid \mathbf{y}\right)}{\sum_{\forall a_{m} \in \mathcal{A}_{l}^{-}} P\left(s_{i}=a_{m} \mid \mathbf{y}\right)} \\
-\underbrace{\ln \frac{P\left(b_{i l}=+1\right)}{P\left(b_{i l}=-1\right)}}_{L_{A}\left(b_{i l}\right)} .
\end{aligned}
$$

It is noteworthy that (21) represents a simple approach of generating the bit-wise extrinsic LLR of $L_{E}\left(b_{i l} \mid \mathbf{y}\right)$, as long as the true symbol APPs of $P\left(s_{i}=a_{m} \mid \mathbf{y}\right)$ can be obtained.

Surprisingly, although we can directly obtain the estimated symbol APPs of $P\left(s_{i}=a_{m} \mid \mathbf{y}\right)$ from the output of the AB-

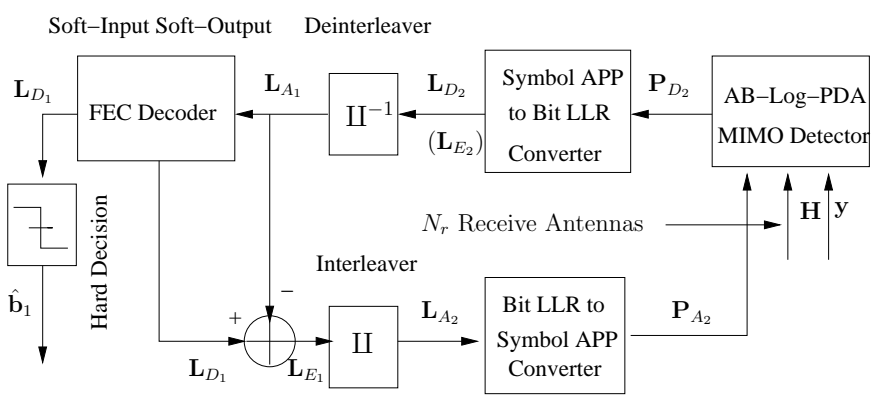

Fig. 2. Simplified structure of the AB-Log-PDA based IDD receiver, where we have $\mathbf{L}_{E_{2}}=\mathbf{L}_{D_{2}}$ rather than the classical $\mathbf{L}_{E_{2}}=\mathbf{L}_{D_{2}}-\mathbf{L}_{A_{2}}$.

Log-PDA, as shown in (16), our study shows that this sort of estimated symbol APPs are not capable of yielding correct extrinsic bit-wise LLRs when invoking $(21)^{1}$. Therefore, the result of (16) should be regarded as "nominal" symbol APPs, rather than the "true" symbol APPs satisfying (21). Below we will show that it is still possible to obtain the extrinsic LLR directly based on the approximate Bayesian Theorem aided symbol APP, which is calculated using (19).

Conjecture 1. The bit-wise extrinsic LLR of the AB-Log-PDA algorithm relying on (16) is given by

$$
L_{E}\left(b_{i l} \mid \mathbf{y}\right) \approx \ln \frac{\sum_{\forall a_{m} \in \mathcal{A}_{l}^{+}} P\left(s_{i}=a_{m} \mid \mathbf{y}\right)}{\sum_{\forall a_{m} \in \mathcal{A}_{l}^{-}} P\left(s_{i}=a_{m} \mid \mathbf{y}\right)},
$$

where $P\left(s_{i}=a_{m} \mid \mathbf{y}\right)$ is calculated by invoking (19).

The $L_{E}\left(b_{i l} \mid \mathbf{y}\right)$ values calculated from (22) using the "nominal" symbol APPs are typically not equivalent to $L_{E}\left(b_{i l} \mid \mathbf{y}\right)$ calculated from (21) using the "true" symbol APPs, but nonetheless, they constitute a good approximation of the latter without inducing any significant performance loss, as it will be demonstrated by our simulations in Section V. As a result, the classic IDD receiver structure of Fig. 1 is simplified to the structure of Fig. 2, where we have $\mathbf{L}_{E_{2}}=\mathbf{L}_{D_{2}}$, rather than $\mathbf{L}_{E_{2}}=\mathbf{L}_{D_{2}}-\mathbf{L}_{A_{2}}$.

\section{Simulation Results ANd Complexity ANAlysis}

In this section, both the performance and the computational complexity of the proposed AB-Log-PDA based IDD scheme are characterized, which further confirms the attractive performance versus complexity tradeoff achieved by the proposed scheme. The FEC employed is the parallel concatenated recursive systematic convolutional (RSC) code based turbo code having a coding rate of $R=\frac{k}{n}=1 / 2$, constraint length of $L=3$ and generator polynomials of $(7,5)$ in octal form. The turbo code is decoded by the Approximate-Log-MAP algorithm using $i t_{t c}=4$ inner iterations. The interleaver employed is the 2400-bit random sequence interleaver. The

\footnotetext{
${ }^{1}$ In fact, if $L_{E}\left(b_{i l} \mid \mathbf{y}\right)$ is calculated by substituting the estimated symbol APPs of $P\left(s_{i}=a_{m} \mid \mathbf{y}\right)$, i.e. the output of the AB-Log-PDA, into Eq. (21), the resultant BER curve of the IDD scheme of Fig. 1 exhibits an anti-bell shape when increasing SNR values. Due to the limitations of space, this BER curve is not presented in this paper.
} 


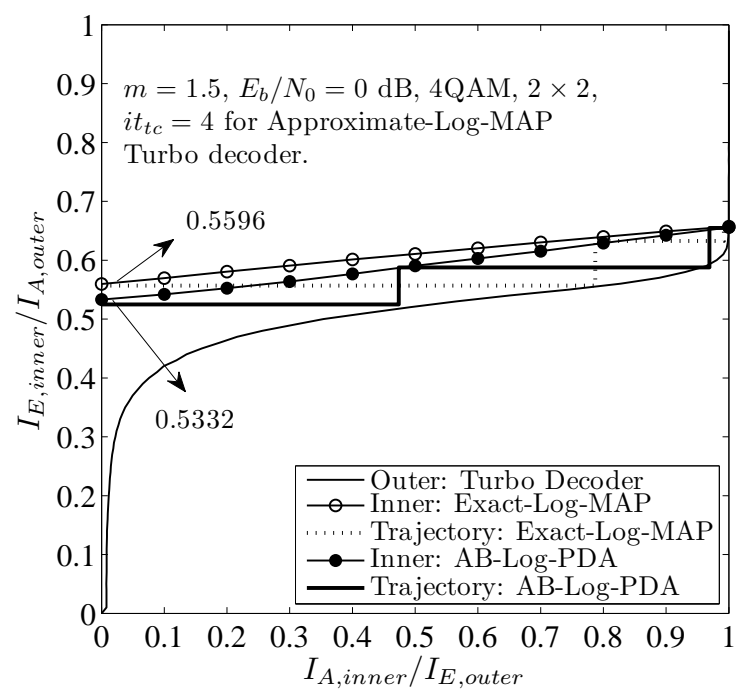

Fig. 3. EXIT chart analysis of the AB-Log-PDA and the Exact-Log-MAP based IDD schemes.

MIMO arrangement is represented in form of $\left(N_{t} \times N_{r}\right)$, and the Nakagami- $m$ fading parameter is denoted by $m$.

\section{A. Performance of the AB-Log-PDA based IDD}

Fig. 3 compares the convergence behavior of the proposed AB-Log-PDA based IDD and that of the optimal Exact-LogMAP based IDD scheme using EXIT chart [12] analysis, where the EXIT curve of the AB-Log-PDA is close to that of the Exact-Log-MAP. For example, when the a priori mutual information is $I_{A \text {,inner }}=0$, the extrinsic mutual information of the AB-Log-PDA and of the Exact-Log-MAP is $I_{E \text {,outer }}=$ 0.5332 and $I_{E, \text { outer }}=0.5596$, respectively. This indicates that the performance of the AB-Log-PDA is close to that of the Exact-Log-MAP in the scenario considered. Additionally, the detection/decoding trajectories indicate that both the AB-LogPDA and the Exact-Log-MAP based IDD schemes converge after three iterations, although the respective performance improvements achieved at each iteration are different.

The above EXIT chart based performance prediction and the convergence behavior of the IDD schemes considered are also characterized by the BER performance results of Fig. 4, where the Nakagami- $m$ fading parameter is set to $m=1.0$, which corresponds to the Rayleigh fading channel. Observe from Fig. 4 that the performance of the AB-LogPDA based IDD scheme is improved upon increasing the number of outer iterations $i t_{o}$, where $i t_{o}=0$ represents the conventional receiver structure in which the signal detector and the FEC decoder are serially concatenated, but operate without exchanging soft information. However, the attainable improvement gradually becomes smaller and the performance achieved after three outer iterations becomes almost the same as that of four outer iterations. This implies that the ABLog-PDA based IDD scheme essentially converges after three outer iterations. A similar convergence profile is also observed for the optimal Exact-Log-MAP based IDD, although its performance is always marginally better than that of the

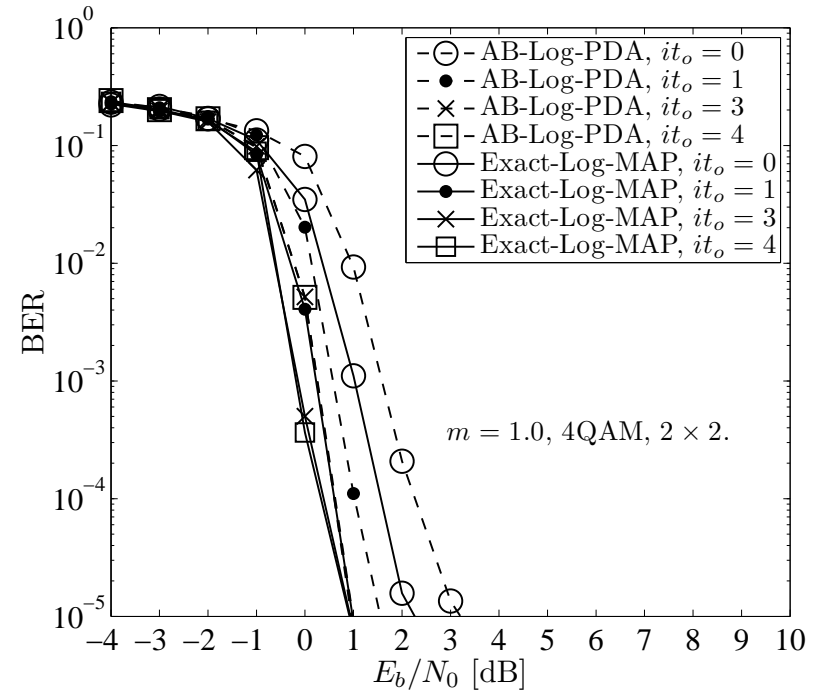

Fig. 4. Impact of the number of outer iterations on the achievable BER of the AB-Log-PDA and the Exact-Log-MAP based IDD schemes.

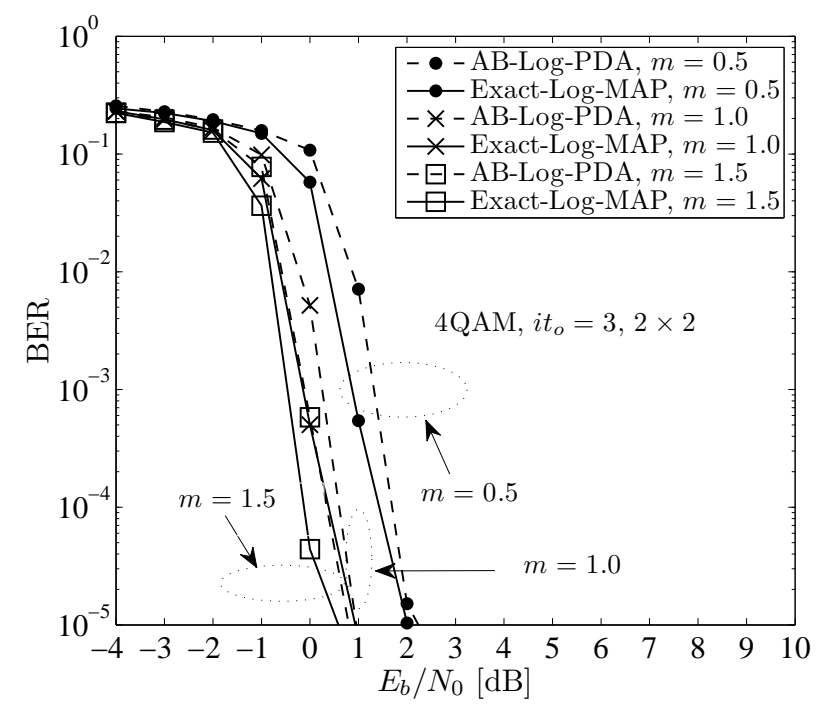

Fig. 5. Impact of Nakagami-m fading parameter $m$ on the achievable BER of the AB-Log-PDA and the Exact-Log-MAP based IDD schemes.

corresponding AB-Log-PDA based IDD. Notably, both IDD schemes considered achieve BER $=10^{-5}$ at about $E_{b} / N_{0}=1$ $\mathrm{dB}$ after three iterations.

Fig. 5 shows the impact of different $m$ values on the achievable BER performance of the IDD schemes considered. As $m$ decreases, the achievable performance of both the IDD schemes considered is degraded, since the fading becomes more severe. However, the performance gap between the ABLog-PDA and the Exact-Log-MAP based IDD schemes is marginal for all values of $m$ considered.

\section{B. Complexity Analysis}

Because the turbo codec module is common to both IDD schemes, and since we have shown that both the AB-Log-PDA and the Exact-Log-MAP based IDD schemes converge after 


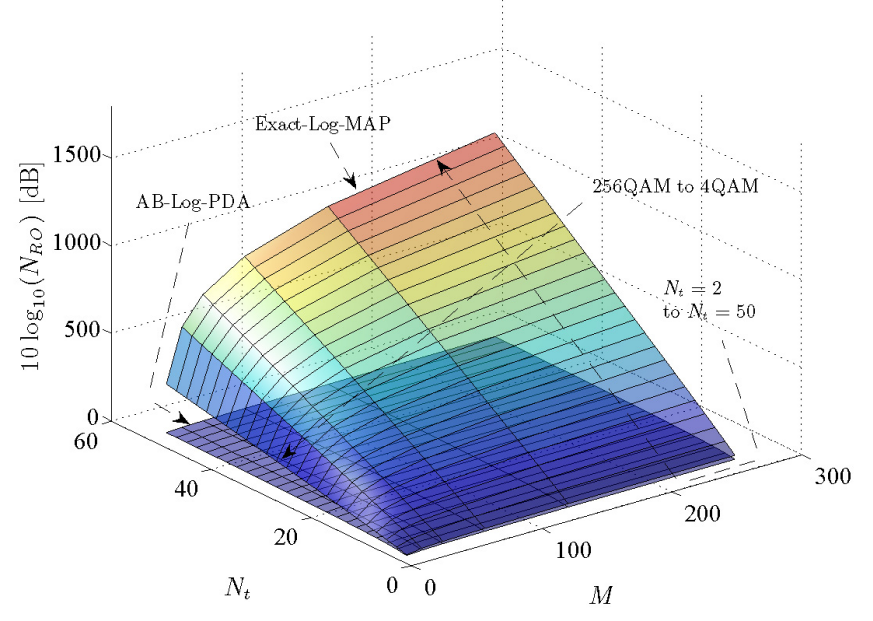

Fig. 6. Computational complexity comparison of the AB-Log-PDA and the Exact-Log-MAP algorithms in terms of the number of real operations $N_{R O}$.

three iterations in the scenarios considered, the computational complexity of the proposed AB-Log-PDA based IDD scheme can be evaluated by simply comparing its complexity to that of the Exact-Log-MAP in a single iteration. As shown in Table II, the major computational cost of the AB-Log-PDA per transmit symbol is the calculation of $\boldsymbol{\Lambda}_{i}^{-1}$ and the matrix multiplication of (13). By using the Sherman-Morrison-Woodbury formula based "speed-up" techniques of [2], the computational cost of calculating $\Lambda_{i}^{-1}$ can be reduced to $\mathcal{O}\left(4 N_{t} N_{r}^{2}\right)$ real operations (additions/multiplications) per iteration. Additionally, the calculation of (13) requires $\mathcal{O}\left(4 M N_{t} N_{r}^{2}+2 M N_{t} N_{r}\right)$ real operations per iteration. In summary, the computational complexity of the AB-Log-PDA method is $\mathcal{O}\left(4 M N_{t} N_{r}^{2}+2 M N_{t} N_{r}\right)+$ $\mathcal{O}\left(4 N_{t} N_{r}^{2}\right)$ per iteration. By comparison, the Exact-Log-MAP algorithm has to calculate the Euclidean distance $\|\mathbf{y}-\mathbf{H s}\|^{2}$ $M^{N_{t}}$ times, hence its complexity order is $\mathcal{O}\left(M^{N_{t}}\right)$. More specifically, $\|\mathbf{y}-\mathbf{H s}\|^{2}$ requires $\mathcal{O}\left(4 N_{r} N_{t}+6 N_{r}\right)$ real operations. Therefore, the Exact-Log-MAP algorithm has a computational complexity of $\mathcal{O}\left[M^{N_{t}}\left(4 N_{r} N_{t}+6 N_{r}\right)\right]$ real operations per iteration, which is significantly higher than that of the ABLog-PDA, especially when $N_{t}, N_{r}$ and $M$ have large values. This observation is further confirmed by the results of Fig. 6 , where the number of real operations is denoted by $N_{R O}$, while considering the scenario of $N_{r}=N_{t}$ as an example.

\section{Conclusions}

In this paper, we showed that the classic relationship where the extrinsic LLRs are given by subtracting the a priori LLRs from the a posteriori LLRs is not valid for calculating the bit-wise extrinsic LLRs of the existing family of PDA based methods when employing $M$-QAM. Therefore, the PDA methods are not as readily applicable to the IDD receiver as they seem to be. This predicament is overcome by our novel approach of calculating the bit-wise extrinsic LLRs conceived for the proposed AB-Log-PDA MIMO detector. Moreover, we showed that the proposed AB-Log-PDA based IDD scheme is capable of achieving a comparable performance to that of the optimal Exact-Log-MAP detector based IDD receiver, while imposing a significantly lower computational complexity in the scenarios considered.

\section{REFERENCES}

[1] M. Breiling and L. Hanzo, "The super-trellis structure of turbo codes," IEEE Transactions on Information Theory, vol. 46, no. 6, pp. 22122228, Sep. 2000.

[2] J. Luo, K. R. Pattipati, P. K. Willett, and F. Hasegawa, "Near optimal multiuser detection in synchronous CDMA using probabilistic data association," IEEE Communications Letters, vol. 5, no. 9, pp. 361-363, Sep. 2001.

[3] D. Pham, K. Pattipati, P. Willet, and J. Luo, "A generalized probabilistic data association detector for multiple antenna systems," IEEE Communications Letters, vol. 8, no. 4, pp. 205-207, Apr. 2004.

[4] S. Liu and Z. Tian, "Near-optimum soft decision equalization for frequency selective MIMO channels," IEEE Transactions on Signal Processing, vol. 52, no. 3, pp. 721-733, Mar. 2004.

[5] Y. Jia, C. M. Vithanage, C. Andrieu, and R. J. Piechocki, "Probabilistic data association for symbol detection in MIMO systems," Electronics Letters, vol. 42, no. 1, pp. 38-40, Jan. 2006

[6] S. Yang, T. Lv, R. Maunder, and L. Hanzo, "Unified bit-based probabilistic data association aided MIMO detection for high-order QAM constellations," IEEE Transactions on Vehicular Technology, vol. 60, no. 3, pp. 981-991, Mar. 2011.

[7] — "Distributed probabilistic-data-association-based soft reception employing base station cooperation in MIMO-aided multiuser multicell systems," IEEE Transactions on Vehicular Technology, vol. 60, no. 7, pp. 3532-3538, Sep. 2011.

[8] J. Proakis, Digital Communications, 4th ed. New York, USA: McGrawHill, 2000.

[9] F. D. Neeser and J. L. Massey, "Proper complex random processes with applications to information theory," IEEE Transactions on Information Theory, vol. 39, no. 4, pp. 1293-1302, Jul. 1993.

[10] T. Adali, P. Schreier, and L. Scharf, "Complex-valued signal processing: The proper way to deal with impropriety," IEEE Transactions on Signal Processing, vol. 59, no. 11, pp. 5101-5125, Nov. 2011.

[11] B. M. Hochwald and S. ten Brink, "Achieving near-capacity on a multiple-antenna channel," IEEE Transactions on Communications, vol. 51, no. 3, pp. 389-399, Mar. 2003.

[12] S. ten Brink, "Convergence behavior of iteratively decoded parallel concatenated codes," IEEE Transactions on Communications, vol. 49, no. 10, pp. 1727-1737, Oct. 2001. 